\title{
NECESIDAD DE LAS HUMANIDADES, SU LUGAR EN LA UNIVERSIDAD
}

\author{
Roxana Pey \\ ICEI y Facultad de Ciencias Sociales \\ Universidad de Chile \\ Pablo Oyarzún \\ Facultad de Artes y Facultad de Filosofía y Humanidades \\ Universidad de Chile
}

A poco andar, casi de inmediato, podrá parecer un poco extravagante nuestro modo de abordar lo que el título enuncia, la "necesidad de las humanidades". Lo hacemos mirando al espacio institucional que está destinado a su cultivo: la universidad; de ahí el subtítulo. Lo hacemos formulando ante todo una pregunta general y por eso mismo inespecífica en lo que concierne a la cuestión de las humanidades, pero cuyo desarrollo, esperamos, dará razón de la pertinencia de la pregunta -o de su ocasión, al menos- para esa misma cuestión.

¿Qué es una universidad? ¿Qué características tiene? ¿Bajo qué condiciones puede y debe ser reconocido un determinado establecimiento como una universidad? ¿Cuáles son los atributos que determinan lo que sea una institución de educación superior y cuáles los que especifican o complementan esos atributos y definen a una universidad? ¿Qué funciones deben ser ejercidas en ella y cómo corresponde que sean institucionalmente fomentadas? Si la universidad es un centro de enseñanza, ¿cuál es el contexto adecuado para el pleno desempeño de la función de enseñar? ¿Satisface una universidad su carácter y misión con la sola docencia y el otorgamiento de grados y títulos? ¿Podría una universidad prescindir de la investigación y seguir correspondiendo a su determinación propia? Y -dejemos esto para más adelante- ¿podría prescindir de las humanidades o concederles solo un papel marginal o complementario en el conjunto de sus tareas? 
No cuesta nada pensar que estas son preguntas teóricas, de alcance meramente especulativo y sin mayores consecuencias, en el mejor de los casos, que contribuir a la formulación de una deseable nomenclatura.

Sin duda, hay innegables implicaciones teóricas en estas preguntas y esas mismas implicaciones, en la medida en que se las aborde, reclamarán con seguridad que se las articule históricamente, es decir, atendiendo a las transformaciones que haya podido experimentar la institución universitaria en el tiempo. Tales implicaciones podrán prestarse a debates probablemente interesantes e indudablemente interesados si de ellas, esto es, de la definición de lo que sea una institución de educación superior y en particular una universidad se siguen consecuencias legales, políticas y económicas.

Es lo que tendría que ocurrir en Chile. Y es que el marco legal que rige para la educación superior en Chile y que sigue reflejando los elementos fundamentales que impuso la reforma de 1981 carece de toda definición de lo que sea una universidad. Lo que la distingue de un instituto profesional, un centro de formación técnica y una escuela matriz de las Fuerzas Armadas, de Orden y Seguridad es el conjunto de licenciaturas obligatorias que aquella ofrezca y cuya obtención establece la ley como condición necesaria para el otorgamiento de determinados títulos profesionales. Esto, y nada más, es lo que legalmente diferencia en Chile a una universidad de un instituto profesional. Parece ser una diferencia muy precaria.

Veamos cómo esta precariedad puede afectar a los imprescindibles cambios que han de ser introducidos en los marcos legales y financieros de la educación superior en nuestro país.

Es previsible que el próximo gobierno -cualquiera que este sea, pues no parece viable ignorar o contravenir el amplio consenso que se ha alcanzado al respecto-resuelva avanzar sustantivamente hacia la gratuidad de la educación superior, probablemente universal, sea con reforma tributaria general, sea con reforma focalizada. Sin embargo, la gratuidad, pieza esencial de una educación que se concibe como un derecho y no como un bien de consumo, no basta para asegurar una efectiva transformación del esquema vigente. Pilar de este es el financiamiento a la demanda. Y este, a su vez, es la pieza esencial y necesaria para generar el "mercado de la educación superior", que en clave característicamente neoliberal la ha regido y regulado en la última treintena. Dejemos a un lado los argumentos decididamente ideológicos que obsesivamente reiteran la libertad de elección para justificar este esquema, a los que no hay más que confrontar con las escandalosas limitaciones de la susodicha libertad que, por eso mismo, no es una, sino varias, condicionadas 
mayormente por el origen socioeconómico de quien "elige" (sin mencionar el derecho a rechazar a determinados "electores" que se reservan algunos establecimientos de la Educación Básica y Media), de manera que buena parte de esas libertades tienden sencillamente a 0 .

El financiamiento a la demanda confirma que la educación no es más que un bien de consumo, como la salud, como la vida misma de quienes jubilan (en este último caso se trata de un mal de consunción más que de un bien de consumo), como cualquiera otra necesidad o antojo de la población que satisfacen el comercio y los servicios. El financiamiento a la demanda tiende a generar un mall de la educación, que además está fuertemente estratificado. De modo que la gratuidad -las etapas que conduzcan a ella-no producirá su efecto si no se cambia el financiamiento a la demanda por el financiamiento a la oferta. En el primero, los "electores" tienen que buscar las instituciones que tengan condiciones de admisión compatibles con las ventajas o desventajas de su formación previa; y aquellas instituciones donde van a dar justamente los que llevan desmedro -no solo por la pobre educación formal que han recibido, sino también por su procedencia socioeconómica, la carencia de relaciones en un país con trazas evidentes de segregación-, aquellas instituciones no ofrecen garantías de prepararlos adecuadamente. Y esto, con el agravante de que el financiamiento a la demanda permite que esas mismas instituciones reciban dineros fiscales a través del crédito sin perjuicio de las fórmulas encubiertas de lucro con que muchas de ellas burlan la ley. En suma, el financiamiento a la demanda tiene como efecto fundamental fortalecer y profundizar la privatización de la educación y completar así la obra iniciada en la dictadura.

Para producir sus frutos beneficiosos, la gratuidad, decimos, requiere el financiamiento a la oferta. Pero este esquema obliga a definir qué es una institución de educación superior y, en particular, la universidad, a fin de determinar las condiciones de elegibilidad de una determinada institución para recibir financiamiento -basal y de desarrollo, bajo una o más de las modalidades posibles- del Estado.

Es, pues, no solo posible sino necesario avanzar a este punto. Pero precisamente en este punto vuelven a incidir las estipulaciones ideológicas. Su principal argumento es el de la diversidad. Pero más que un argumento -y no habría dificultad en someterlo a discusión en cuanto tal- es un subterfugio: pues ¿qué cubre o que posibilita el principio de la diversidad? Supuestamente atiende a la diversidad social, económica, cultural, confesional, etc., de la población; y ella se ve reflejada en la diversidad de propiedad, de misión, de 
proyecto, de estructura, organización y funcionamiento de las universidades. Y en parte es así: hay diversidad en cuanto a instituciones públicas y privadas, laicas y confesionales, socialmente elitarias o heterogéneas, de amplia cobertura disciplinaria o focalizadas, etc. Y si uno examina los cruces que hay entre estas distintas características -y aquellos que no se producen-, se comprobará que la reivindicación del principio de la diversidad tiene un fondo de segregación evidente.

Lo que parece ser la distinción más aceptada en lo que toca a la diversidad, al margen de la propiedad, la orientación o el espectro social, analizadas las universidades en su condición de instituciones de conocimiento, es aquella que las divide entre complejas y simples, es decir, estas últimas, docentes. Desde este específico punto de vista (que no tiene nada de puro, porque se mezcla con las anteriores características), se podría decir que el prurito de la diversidad viene a dar a esto, a la justificación de la existencia de instituciones que ofrecen títulos y grados como único producto.

Dicho sin rodeos, esta es una de las aberraciones de la reforma de 1981, no tanto porque sean del todo inconcebibles unos establecimientos de educación superior meramente docentes, sino porque permitió que se crearan instituciones que se arrogaran el nombre de universidades bajo el supuesto de que la sola docencia bastaba a su definición.

Permítasenos una anécdota al respecto: uno de los autores de este artículo, aquejado de cesantía a su regreso del extranjero en aquel año tan preñado de posteridad para la educación chilena, fue a dar al recinto de una naciente universidad -la circunstancia que hizo posible esa visita no requiere ser referida aquí- y a la oficina de su incipiente rectora. La oficina estaba habilitada con sillones y mesas del jardín de lo que había sido la embajada de un país socialista. Cuando la escueta conversación derivaba hacia la concepción que habría animado la enseñanza del profesor cesante en las futuras aulas de los estudiantes de leyes, sonó el teléfono. De lo que el profesor escuchó pudo inferir que se trataba de la malla curricular de la carrera de derecho; abreviando, el intercambio concluyó con una declaración tajante de la rectora: "no, no puedes reducir la carrera a seis semestres". La otra conversación, del profesor con la señora en cuestión, referida a lo que sería la base de enseñanza de la filosofía del derecho que aquel impartiría, se cerró con otra declaración contundente de la señora: "naturalismo, por cierto"; el profesor, para salir del paso, replicó: "bueno, qué lástima, yo soy positivista". Nos reservamos revelar de qué universidad se trataba por no mancillar el nombre de nuestra premio Nobel. 
Mientras eso ocurría, en otro lugar de la capital, donde la principal universidad soportaba feroces embates, y precisamente en el que sigue siendo su campus más golpeado, se hacía sentir de otro modo la ley del 81: las licenciaturas se recortaban de 5 a 4 años; a los estudiantes afectados por esta abreviatura que tuviesen avanzadas sus investigaciones se les ofreció convalidarlas por el grado de magíster. La co-autora de este artículo recuerda a un heroico joven de apellido Medina que rechazó esa oferta y exigió que le otorgasen solamente el grado de licenciado por una tesis que a todas luces era excelente. Su sacrificio, si podemos llamarlo así, fue, en ese momento, la única resistencia a la brutal intervención, y no es improbable que lo haya motivado una intuición certera del inicio de la privatización y la aguda precarización que la ley traería inevitablemente consigo. El episodio casi rima con las recientes tentativas de aplicar nuevos recortes a la duración de las carreras sin ninguna consideración de condiciones previas de posibilidad ni consecuencias; digamos que se sigue buscando ayudar al mercado mediante el abaratamiento de costos.

Son anécdotas, claro, incidentes aislados se podrá pensar; el punto es que se podrían sumar muchos más hasta hacer visible en ellos, a la manera de síntoma o de primicia, el paradigma de la universidad docente a la medida de la legislación de la dictadura y de la mercantilización que ella ha inducido. Y es cierto que bien poco se ha hecho por no diremos rectificar (porque tal cosa no es posible), sino derechamente abolir ese paradigma. En esas primicias se advierte a plena luz el fraude que han sido y son hasta hoy aquellas que, entre las muchas instituciones que se crearon al amparo inexpugnable de la reforma dictatorial, no han pasado de ser sedicentes universidades.

El tema tiene también su interés por lo que veníamos diciendo: no es del todo inconcebible que existan universidades docentes a condición de que se entienda que no son universidades por sí mismas, sino solo en la medida en que completan lo que les falta con el concurso de capacidades provenientes de otras universidades, que son complejas. Desde luego un aserto como este descansa sobre un supuesto: que la transferencia del conocimiento (hablemos en estos términos, para simplificar) es inseparable de la generación de conocimiento. Y no parece que este sea un supuesto atrabiliario. Por cierto, podemos agregar más elementos a la idea de la complejidad. Pero si solo se habla de docencia, parece necesario conceder que la complejidad ya está implicada en ella, ya está contenida analíticamente en la relación entre generación y transferencia en la diversidad de niveles formativos, ya por lo dicho, porque la docencia de las "universidades simples" requiere capacidades que no pueden ser 
meramente reproductivas, ya porque el postgrado encarna esa relación, al no ser posible sin capacidades instaladas, efectivas y activas de generación del conocimiento. Y no mencionemos aquí la extensión, la vinculación con el medio, la inserción en la sociedad y otros rasgos que suelen atribuirse a la institución universitaria.

Es una evidencia insoslayable del pobre sistema de acreditación de instituciones de educación superior con que cuenta el país el hecho de que su obligatoriedad se restrinja a la docencia (de pregrado) y a la gestión institucional. Ella refuerza la idea de que la docencia basta a la definición de una universidad, aunque se subentienda que se está hablando del mínimo y que en las demás dimensiones que se agreguen a este mínimo se mide el nivel de complejidad que es dable atribuir a una institución dada. La complejidad es una añadidura que en el mejor de los casos permite distinguir entre instituciones por la variedad de funciones que abarcan (sin que ello implique discernir calidad), pero ya la sola docencia es el umbral a partir del cual hay universidad. Demás está decir que este es uno de los aspectos en que es preciso reformar de la manera más radical nuestro sistema de acreditación, además de lo que atañe a su evidenciada vulnerabilidad.

La docencia, como función esencial de la universidad, requiere, pues, de un entorno de investigación y debate, ya sea que este se presente en una misma institución (y solo en este caso podríamos hablar de universidad), ya sea que lo aporte el sistema. Pero, por cierto, para que el sistema se lo aporte a aquellos institutos cuya oferta exclusiva es docente, se tienen que producir por fuerza desequilibrios. No cuesta mucho comprobarlos observando el nomadismo académico que en muchos casos induce un esquema restrictivo de remuneraciones y que en Chile se viene dando desde la época de la dictadura. En estos casos, las universidades meramente docentes gozan del subsidio que les prestan algunas de las complejas mediante el concurso de académicos que han desarrollado capacidades investigativas y que buscan suplementar sus bajas remuneraciones con los emolumentos que reciben de esas instituciones a cambio de prestaciones docentes. La fórmula es perversa y en ninguna otra área se manifiesta más gravemente que en aquella de las llamadas "disciplinas blandas": las humanidades, las ciencias sociales y las artes.

Sigamos esta cuerda para abordar ahora la cuestión de las humanidades. Concedida la relación inseparable de investigación y enseñanza en la educación superior, sobresale un aspecto interesante que las toca derechamente. Piénsese en aquellas universidades (que haya en total 57 en el país -descontada la Universidad del Mar- y que todas sean complejas parece imposible, quién 
podría negarlo, pues las capacidades instaladas no son infinitas) que cifran su opción en uno o más focos caracterizados porque conllevan rentabilidad en la medida en que preparan "capital humano avanzado" (para emplear la jerga internacional que repite CONICYT) en vista de desempeños que están vinculados al desarrollo productivo del país (donde "producción" tiene necesariamente un rédito contable) o también de actividades y servicios que son imprescindibles para ese desarrollo. Piénsese en universidades que vinculan su enseñanza a la investigación e incluso despliegan (y no solo por razones de marketing, aunque estas igualmente cuentan) lo que hoy se llama vinculación con el medio en el viejo sentido de extensión cultural. Salvo contadas excepciones (que habría que examinar más de cerca), las humanidades, en la medida en que estén presentes, comparecen en ellas bajo la forma exclusiva de la docencia, sirviendo lo que se suele llamar -y de manera muy laxa- "formación general".

Y esto constituye un dato - o síntoma, si se quiere- del peso específico que se les reconoce. Es cierto: lo observamos en instituciones de las características que recién mencionábamos. Pero estos datos se convierten en síntomas cuando se examina lo que ocurre no solo en otras universidades que aspiran al apelativo de complejas en toda su magnitud, sino en el sistema estatal de fomento a la investigación e innovación.

En lo que toca a las universidades, algunas de ellas pueden concederle a las humanidades una significación específica (nunca central) por razones ideológicas, es decir, confesionales. Se podría hasta pensar que algunas instituciones privadas que posibilitó la ley de la dictadura (no se olvide nunca este origen) podrían atribuirle más importancia aún si así lo determina el capricho de sus dueños ${ }^{1}$. Pero también en la principal de la universidades públicas se ha requerido de medidas excepcionales (y transitorias) para justificar la validez de la inversión que pueda hacerse en ellas. En este caso, por muchos argumentos que se enarbolen para convencer a un interlocutor que de antemano está convencido de lo contrario, ninguno de ellos alcanza para hacerle la menor mella a las políticas públicas.

\footnotetext{
Hay que reconocer que esta última observación excede su marco y soslaya un punto importante: que las universidades privadas de mayor monta le atribuyen a la presencia y cultivo de las humanidades en sus claustros una importancia que no puede ser simplemente achacada a causas de un modo u otro incidentales. Es que -de manera más o menos explícita-se tiende a ver en esa presencia y cultivo aquello de lo que no podría prescindir una universidad sin dejar de serlo. Y vale la pena retener este síntoma para lo que sigue.
} 
Cualquiera que se asome a lo que en este último terreno se promueve verá que las humanidades no constituyen prioridad en ningún sentido atendible. En las estadísticas que presenta FONDECYT para los años 2008 a 2012, las humanidades alcanzan a un $10,11 \%$ en proyectos de iniciación, 7,60\% en postdoctorado y $10,97 \%$ en proyectos regulares ${ }^{2}$. Esta es, entonces, la gravitación que tienen las humanidades en las políticas de investigación del país: y por cierto no hay otros instrumentos para estimular de manera sustantiva la pesquisa en humanidades.

Luego, al examinar las condiciones de validación que se aplica a esta pesquisa se hallará una notoria tendencia a uniformar lo que sea pertinente para las humanidades con lo que rige para las ciencias. Y la tendencia se ha agudizado. Así, es requisito expreso de aprobación de un informe final de investigación la publicación de al menos un artículo en revista indexada (Thomson-Reuters, es decir, ex-ISI da la norma y hay equivalencias -limitadasque se espigan de Scopus, Scielo y Latindex Catálogo); un libro o un capítulo de libro se registra en "Otras Publicaciones" y no se incluye en el requisito de aprobación: se lo admite en esta calidad previa demostración de que ha sido sometido a un arbitraje externo. La discusión en torno a los pesos relativos de un artículo en revista indexada y de un libro es ya de larga data. En filosofía, para los efectos de la evaluación curricular, se le asignan 12 puntos a "una ISI", como se dice familiarmente, y 20 a un libro; digamos que esta escueta diferencia de 8 puntos ha sido un logro que no se obtuvo expeditamente y por cierto que a la hora de justificar con precisión el quantum (los dichosos 8 puntos) no hay razón que atine.

En un número reciente de esta misma publicación, José Santos hablaba persuasivamente de "la tiranía del paper", confrontando la normalización del conocimiento que este producto refleja con la diversidad de discursos que es propia de las humanidades ${ }^{3}$. Y el asunto, sin duda, no es menor. Para un investigador de las humanidades, un libro -y en su forma más alta, por anacrónico que suene, una obra- es el producto principal del conocimiento, que probablemente ha de tener como afluentes unos artículos, los cuales, en este preciso caso, no adolecen de la transitoriedad que es la regla de los

2 Las ciencias sociales presentan las siguientes cifras en los respectivos rubros: $21,82 \%, 3,91 \%$ y $18,56 \%$.

José Santos Herceg, “Tiranía del paper. Imposición institucional de un tipo discursivo", en: Revista Chilena de Literatura, 82 (2012), 197-217. 
papers. Para ese investigador no son indiferentes lenguaje y escritura (es decir, estilo), que los papers allanan a la condición de meros vehículos, con el agravante de imponer una suerte de inglés de la koiné escolar como única lengua válida o como modelo obligado.

Tal como se podrá verificar, ya se ha argumentado con fuerza y con pruebas al respecto. Pero valdría la pena insistir sobre la cuestión de la escritura y la diversidad discursiva para referirnos al estatuto del ensayo y su estrecha vinculación con el desarrollo de las humanidades en América Latina. Sin duda, podemos hablar también de otras latitudes y en términos similares, pero esta significación nos concierne de manera esencial. Y es que en nuestras regiones el ensayo es un tipo de escritura privilegiado en la producción, comunicación y circulación de conocimiento desde los comienzos de la época republicana. Por cierto, se dirá que la índole dubitativa, problemática, digresiva e inconcluyente del ensayo no se aviene con lo que es exigible del conocimiento, es decir, con la fuerza demostrativa y probatoria; allá más bien se trata de pensar, no de conocer. Pero precisamente esto le confiere al ensayo (y a las formas de escritura que le son afines) una suerte de eminencia. No es que se discuta la posibilidad de conocer en el ámbito de las humanidades, en el sentido demostrativo de la palabra; por supuesto que hay conocimiento en ellas, tesis, demostraciones y pruebas. Pero el punto es que las humanidades operan en un campo que no está dado ni determinado en su legalidad y configuración: lo humano no es un dato para ellas, sino un problema. En esa medida, la diversidad discursiva que las caracteriza -su resistencia a la estandarización y a la retórica uniforme del paper, resistencia que tiene precisamente en el ensayo su manifestación más visible- da cuenta de algo más que una porfía por refugiarse en viejas formas: expresa una apertura en la que se hace particularmente perceptible la pregunta por lo humano en su tenaz indeterminación.

Y si de todos modos se quiere ponderar la significación o función del artículo en las humanidades, se tendrá que reconocer que tiende (al menos tiende) a hacer en ellas una figura distinta. Hay un punto ideal en que los artículos en torno a un determinado asunto son entre sí contemporáneos a pesar de las distancias temporales que puedan separarlos y aunque se conozca bien o se pueda reconstruir la adscripción de cada uno a cierta escuela u orientación y muchas de estas se encuentren perimidas. Hay un punto ideal en que siguen siendo vigentes, a la medida de la vigencia del asunto del cual se ocupan. En esta región del saber, consultar un viejo artículo sobre la cosa que interesa al investigador de hoy (en literatura, filosofía, historia, por ejemplo) puede resultar muy instructivo y, aun más, revelador. 
Son notorias las restricciones impuestas a las humanidades, a su ejercicio y desarrollo, por las formas dominantes del diseño, la organización y el financiamiento actual del trabajo académico, y por los criterios y requerimientos bajo los cuales se evalúa y reconoce el aporte de las disciplinas y de la investigación que se realiza en ellas. Con ser sensiblemente estrechas, estas restricciones no son inocuas. Basta para ello confrontar las características y condiciones propias de las humanidades con los parámetros que articulan hoy todo el trabajo científico, teniendo, por supuesto, al par (cada vez más indiscernible) de ciencia-y-tecnología (de tecno-ciencias) como eje: productividad, rendimiento, eficiencia, emprendimiento e innovación y su soporte y, a la vez, su expresión en el "capital humano avanzado". Todo ello es tributario de lo que habría que llamar el modo de producción del conocimiento imperante en la época del capitalismo tardío: esos mismos parámetros son clara expresión de este último, así como la "sociedad del conocimiento" no significa otra cosa que la total subordinación del conocimiento a las necesidades y exigencias de totalización del capitalismo.

No debiera extrañar, entonces, que en los últimos años se haya encendido el debate en torno a las humanidades y su importancia. Es bien conocido el inteligente alegato de Martha Nussbaum, que tiene en su libro Sin fines de lucro ${ }^{4}$ la pieza ejemplar. Centro del debate es precisamente la diferencia que hacen las humanidades respecto de los imperativos unilaterales y obsesivos del crecimiento económico, diferencia que precisamente les acarrea ostensible desventaja en el esquema vigente que tiene a ese crecimiento como ley irrebasable. $\mathrm{Y}$ es que esa diferencia pone precisamente en cuestión, y de manera radical, la primacía de lo económico, en el sentido restringido (y a la vez global) que es propio del capitalismo y muy especialmente de su forma tardía.

A su vez, se afirma (lo hace enfáticamente Nussbaum) que la contribución que las humanidades entregan a la democracia es esencial e insustituible. Seguramente se puede argumentar que las competencias que son necesarias para el crecimiento económico, si no son las mismas que favorecen el cultivo de las humanidades, bien pueden ser fortalecidas o enriquecidas por estas últimas, un poco en el sentido de que productores que viven una 
vida de horizontes más amplios producen mejor, o bien que la versatilidad y las destrezas de comprensión de situaciones complejas que promueven las humanidades son capacidades valiosas y a veces determinantes en los medios en que se desenvuelve la labor económica de punta, lo que es ya un viejo hallazgo de las empresas.

Pero un argumento de esta naturaleza - que no es falso-se limita a solicitar el favor del soberano, que se reserva la decisión. Lo democrático de las humanidades estriba en la impugnación por principio de esa soberanía y de esa decisión y apunta, por eso mismo, a aquello que en lo humano es la potencia de lo común; y nuevamente habrá que advertir: no lo común como algo dado, sino como algo en el proceso de cuya (interminable) gestación se constituye (también interminablemente) lo humano: en la potencia de pensar más allá de los privados intereses (en eso consiste, a fin de cuentas, pensar), en la potencia de interesarse por otras vidas y por el espesor histórico que traen consigo, en la potencia de abrirse a la complejidad del mundo y de la existencia, en la potencia, en fin, de dejarse afectar por lo diverso y comprenderlo. En este sentido, lo democrático de las humanidades es inseparable de considerar lo humano como tarea de construcción común que tiene su fin en sí misma y su indicador esencial en la índole incalculable de lo humano mismo; por eso no pueden concebir lo humano como capital, en cualquiera de sus formas (y por "avanzado" que pueda ser), sino como un acontecimiento y un advenimiento que no cesa de acontecer y no termina de advenir, pura posibilidad que de ningún modo (o solo negándola) es capitalizable.

Las humanidades no tienen su foco en la producción, sino en la creación. ¿Qué quiere decir esto? No se trata de reintroducir en la discusión valores incontrolables con tufillo teológico: se trata de indicar la diferencia entre dos procesos materiales y sus respectivas reglas. La producción se mide por su resultado y su rédito, de manera que la conmensurabilidad entre el trabajo que se invierte en ella junto al tiempo que supone ese trabajo, por una parte, y, por otra, el producto que de ello resulta junto al retorno que conlleva es lo que determina la producción como tal: su estructura es teleológica y económica. Se suma a esto decisivamente que la referida conmensurabilidad asegura o por lo menos posibilita la replicación del proceso y una vez establecida ya no es necesario volver a ensayar operaciones para obtener el mismo resultado. En consecuencia, el éxito teleológico del proceso (el logro del resultado) no tiene solo efecto económico en cuanto a rédito, sino también en cuanto al proceso mismo y al tiempo que requiere. 
En cambio, la creación (en un sentido amplio del término, no limitado a la labor artística con la que generalmente se la asocia) tiene su medida en la variación, en la emergencia de la singularidad a la que se abre y que busca sin certeza ni garantía. Es trabajo, sin duda, concreto y material, que no carece de reglas y de operaciones aprendidas: desde este punto de vista nada parece diferenciarla de la producción y de hecho comparte con ella todos los elementos y las fases que se quiera mencionar. Y sin embargo su medida es lo que interrumpe y rompe la conmensurabilidad. Por cierto, nada asegura que ocurra esa interrupción: en la contabilidad de la creación no es posible proyectar un haber, solo cabe presuponer un debe hasta que el tiempo, dado el caso, diga lo contrario. Por eso mismo, la variación que persigue el proceso creativo (y que solo puede darse a condición de no acosarla, de no buscarla donde se cree que está) no tiene un tiempo prefijado ni previsible de presentarse y es perfectamente verosímil, en definitiva, que no se presente. La temporalidad de la creación es la del diferimiento, tanto, cuanto sea necesario para que se dé la variación: que puede no darse en absoluto. De ahí que en la economía de la creación (economía que le es inseparable, como es inseparable a todo lo humano) hay un núcleo an-económico ${ }^{5}$.

Esto explica el problema que ellas presentan ante los indicadores que rigen hoy por hoy la gestión universitaria y que tienden a asimilarla a la gestión empresarial. Si no se cuenta ni siquiera con indicadores de rentabilidad social (y esto todavía es mantenerse dentro de la lógica del capitalismo tardío), para qué hablar de aquello que es precisamente reacio al tipo de medición que implican esos indicadores maestros. La matriz y supuesto de estos es la estandarización; en las humanidades lo que cuenta es la variación y la divergencia. No se trata, entonces, de rechazar de plano la aplicación de los juegos usuales de indicadores que dan cuenta del cumplimiento o incumplimiento de estándares y metas en rangos de inferior a superior a

\footnotetext{
Y habría que decirlo: este núcleo es lo humano mismo, aquello que llamamos lo "humano" sin poderlo traer nunca a la horma de una definición o de una forma enteramente estabilizada. Lo "humano" en nosotros, que ciertamente se manifiesta y expresa de manera originaria en la producción, nunca queda completamente determinado por ella, así como ella no agota ni absorbe jamás del todo el tiempo en que se despliega lo "humano", y este vuelve a hallarse con su mero hecho de ser precisamente en los momentos en que la conmensurabilidad económica se rompe, en la fiesta, en el goce, en el hallazgo, en la muerte. Son momentos que enseñan que lo "humano" no es capitalizable: que es un acontecimiento en última instancia gratuito.
} 
propósito de una determinada disciplina, una organización o una actividad académica. No se trata de eximirse de toda normalidad del conocimiento. Declarar una excepcionalidad irreducible bien puede servir al contrabando de cosas académicamente indeseables que se escudan bajo esa suerte de inmunidad ante cualquier tipo de medición con el alegato de lo excepcional o lo especial que en ocasiones esconde mera mediocridad. Se trata de atender a la variación, se trata de entender que en la divergencia se juega, precisamente, eso que llamamos "creación": inventiva (en el sentido primario de hallazgo y ocurrencia), generación de nuevo conocimiento o de nuevas estrategias de conocimiento, desplazamiento de fronteras epistémicas, gestación y cuidado de la "obra".

Por cierto, no estamos afirmando que las humanidades sean el único tipo de conocimiento, de disposición reflexiva e investigativa que exhiba estas características. Así como hay normalidad y mera reproducción en ellas y bajo las condiciones vigentes quienes las cultivan en las universidades se ven forzados a adaptarse a las exigencias de la escolaridad contemporánea (cada vez más, con el agravante de que a veces no hallan mucha dificultad en ello y hasta se precian de hacerlo), en las otras disciplinas, especialmente las científicas, se da lo que se aparta del estándar y resiste los criterios de homologación. También en ellas lo que esencialmente cuenta es el vector divergente de la creación, porque, al fin y al cabo, este es, no el indicador, sino el índice, la señal, el síntoma: la primicia del conocimiento -del pensamiento.

Solo que hoy las humanidades conservan la memoria de algo que cabría llamar propiamente un estilo que tiende a ser obliterado aceleradamente en las prácticas universitarias y formativas actuales, incluso en las que les son más propias a ellas mismas. Mientras por doquier se impone el imperativo del conocimiento no por sí mismo, sino condicionado a resultados, y las humanidades también parecen llevadas a rendírsele, lo que en ellas se hace no termina jamás de suprimir dos rasgos que les son esenciales: el estudio y la conversación. El estudio es celo, cuidado, ardor y pasión y esencialmente atención. La atención es acogida de las singularidades, de lo que se resiste a ser homologado, catalogado, categorizado, reducido a estándares y administrado según estos. Y la conversación consiste en dos cosas: en el trato frecuente con esas singularidades, que acostumbra a quien así se conduce a aprender de ellas precisamente lo que las singulariza; y consiste la conversación también en solicitar de los otros similar atención y trato y compartir con ellos lo que de ese modo se aprende. 
Si miramos lo que decíamos a propósito de la universidad y de la inseparable relación entre investigación y docencia, podrá advertirse por qué importa fomentar el cultivo de las humanidades en los institutos superiores, por qué es importante, asimismo, alentar a las y los jóvenes que estudian humanidades a que en ellas y ellos se desarrolle el sentido esencial de ese cultivo sin que queden del todo cautivos del aparato académico contemporáneo al que tienen que responder por fuerza si quieren hacer carrera en él: no quedar del todo cautivos para no convertirse sin más en algo así como ejecutivos del conocimiento. Se advertirá también por qué es importante garantizar que todas y todos los estudiantes alcancen un grado de familiaridad con lo que hace un momento llamábamos su estilo, sus diversos modos de aproximarse y abordar sus asuntos, la atención a lo singular y lo diverso, el cuidado de las formas de comunicación, en fin, esa tarea peculiar (se podrá creer que hasta paradójica y en todo caso eminentemente política) de vincular lo irreducible a lo común.

Las humanidades son, en la universidad, la insistencia de una disposición y una tarea que está en el fundamento mismo de lo universitario, si hemos de entender este último como la voluntad no condicionada de saber y de saber en común en pro de lo común: dicho de otro modo, como la incondicionalidad del conocimiento en indiscernible vinculación con la apertura de lo humano.

Esto es lo que las humanidades aportan a la universidad como institución, magnitud histórica y modo de vida. Por eso la cuestión de las humanidades es indisociable de la cuestión de la universidad.

Los tiempos venideros del país estarán marcados, como el presente, no exclusivamente, por cierto, pero en una medida indudablemente significativa y urgente, por los temas de la educación en todos sus niveles y en todos sus ámbitos. Los debates, decisiones e iniciativas que de ello se desprendan marcarán, a su vez, el futuro del país y de su población. Son, sin duda, muchos los temas que están en juego, si lo que se quiere es una educación que no sea solo un instrumento al servicio del crecimiento económico y de la profundización del modelo vigente como normas supremas, con sus consecuencias inevitables de segregación e injusticia, sino como un espacio de desarrollo humano y de formación de ciudadanía. Esos temas tendrán uno de sus ejes en la gratuidad. A partir de lo dicho aquí, lo que se puede decir al respecto es que la gratuidad no solo supone el reconocimiento de la educación como un derecho social universal e irrenunciable que el Estado debe garantizar, favoreciendo la igualdad, no solo implica también condiciones de paridad para el desarrollo de aquellas disciplinas que no cifran lo esencial de 
sus tareas y aportes en el rendimiento económico, sino que también toca algo a lo que aludimos pasajeramente en una nota, casi como en sordina: algo que precisamente concierne a las humanidades en su principio, en cuanto que le hablan ellas al ser humano en su incertidumbre y su apertura y en lo que se podría llamar su propia, primaria e íntima gratuidad. 